1 Universidade Regional do Noroeste do Estado do Rio Grande do Sul (Unijuí). Departamento de Ciências da Vida - ljuí (RS), Brasil. tais.liberalesso@gmail.com

2 Pontifícia Universidade Católica do Rio Grande do Sul (PUCRS) - Porto Alegre (RS), Brasil.

fer_dallazen@hotmail.com

3 Universidade Regional do Noroeste do Estado do Rio Grande do Sul (Unijuí). Departamento de Ciências da Vida - ljuí (RS), Brasil. vanessa.acbandeira@yahoo. com.br

4 Universidade Regional do Noroeste do Estado do Rio Grande do Sul (Unijuí). Departamento de Ciências da Vida - ljuí (RS), Brasil. evelise@unijui.edu.br

\section{Prevalência de fragilidade em uma população de longevos na região Sul do Brasil}

\author{
Prevalence of frailty in a long-lived population in the Southern region \\ of Brazil
}

Taís Elizabete Manfio Liberalesso', Fernanda Dallazen², Vanessa Adelina Casali Bandeira ${ }^{\mathbf{3}}$, Evelise Moraes Berlezi ${ }^{4}$

RESUMO Objetiva investigar a prevalência de fragilidade em uma população de longevos. Estudo observacional e transversal com idosos acima de 80 anos de um município da região Sul do Brasil. A síndrome da fragilidade foi identificada de acordo com Fried et al. (2001). Entre os 69 idosos, 58\% eram frágeis e $42 \%$ pré-frágeis, com maior frequência de diminuição de velocidade da marcha, perda de peso e fadiga. É evidente que a fragilidade está instalada em idosos longevos, assim, avaliar e classificar os idosos de acordo com a fragilidade possibilita a organização das prioridades de assistência, com o objetivo de prevenir, manter e/ou restaurar a capacidade funcional.

PALAVRAS-CHAVE Idoso de 80 anos ou mais. Idoso fragilizado. Envelhecimento.

ABSTRACT It aims to investigate the prevalence of frailty in a long-lived population. Observational and cross-sectional study with elderly people over 80 years old in a municipality of the Southern region of Brazil. Frailty syndrome was identified according to Fried et al. (2001). Among the 69 elderly, $58 \%$ was frail and $42 \%$ was pre-frail, with higher frequency of slowing of gait speed, weight loss and fatigue. It is evident that the frailty is installed in long-lived elderlies, therefore, evaluating and classifying the elderly according to a frailty makes it possible to organize care priorities, with the aim of preventing, maintaining and/or restoring functional capacity.

KEYWORDS Aged, 80 and over. Frail elderly. Aging. 


\section{Introdução}

Com o aumento da expectativa de vida da população, a proporção de idosos com 80 anos ou mais aumentou consideravelmente, o que resulta em um fenômeno de grande repercussão no Brasil, uma vez que a longevidade se reflete em todas as dimensões e setores da sociedade (PEREIRA ET AL., 2015). Atualmente, essa faixa etária representa $1,5 \%$ da pirâmide etária brasileira e 1,6\% da região Sul (BRASIL, 2012).

O processo de envelhecimento resulta das consequências da passagem do tempo, que são progressivas, irreversíveis e promovem comprometimento dos principais sistemas fisiológicos. Essas mudanças relacionam-se a danos moleculares e celulares, decorrentes do declínio geral da capacidade intrínseca do indivíduo, manifestando-se de forma singular e sendo mais evidentes em idades mais avançadas (KALACHE, 2008).

É fundamental, nesse sentido, avaliar aspectos do envelhecimento, entre eles, a fragilidade, que é uma síndrome multidimensional. De acordo com Fried et al. (2001), a síndrome da fragilidade é definida pelo declínio de energia e está relacionada com alterações fisiológicas dos sistemas musculoesquelético, neuroendócrino e imunológico, que repercutem, principalmente, na perda de massa muscular; na alteração de apetite; e no estado inflamatório crônico. Dessa forma, o fenótipo de fragilidade envolve cinco fatores: perda de peso, fadiga, fraqueza muscular, inatividade física e lentidão da marcha. De acordo com os autores, classifica-se como idoso frágil aquele que apresenta três ou mais desses componentes; pré-frágil na presença de um ou dois componentes; e não frágil na ausência de componentes.

Ainda, a síndrome da fragilidade está associada a fatores físicos, cognitivos, sociais, econômicos e ambientais. Embora a fragilidade seja uma condição fisiológica, ela agrava-se na presença e número de doenças associadas. Essa condição aumenta o risco de comprometimento da capacidade físico-funcional, de complicações de doenças, ocorrência de quedas, institucionalização, hospitalização e morte (FRIED ET AL., 2004; VERMEIREN ET AL., 2016).

Apesar de ser uma condição progressiva, a síndrome da fragilidade apresenta possibilidades de prevenção e reabilitação. A prevenção deve ser uma tarefa dinâmica e necessária para melhor efetividade. Para tanto, a identificação de fenótipo de fragilidade nas populações, especialmente nas vinculadas às Estratégias Saúde da Família (ESFs), permite a adoção de medidas de intervenção específicas, que contribuem para a organização da demanda, com priorização dos idosos com fragilidade instalada, o que abre um portfólio de possibilidades de intervenção individuais ou coletivas, tanto em idosos frágeis como não frágeis (TEIXEIRA; NÉRI, 2006).

Dessa forma, é fundamental a aplicação de instrumentos confiáveis que possam ajudar na identificação de idosos frágeis e potencialmente frágeis para a individualização da assistência. Diante dessas considerações, o presente estudo tem como objetivo investigar a prevalência de fragilidade em uma população de longevos de um município da região Sul do Brasil.

\section{Métodos}

Este estudo é de delineamento observacional, transversal e não probabilístico. A população do estudo é composta de idosos octogenários, nonagenários e centenários, de ambos os sexos, residentes na área urbana do município de Erval Seco, localizado na região do Alto Uruguai, no estado do Rio Grande do Sul.

$\mathrm{O}$ referido município, segundo censo do Instituto Brasileiro de Geografia e Estatística (IBGE) de 2010, apresentava uma população de 7.878 habitantes, dos quais, 3.920 eram homens e 3.958 mulheres. Com relação a idosos longevos, o município apresenta 1,98\% (156) da população com idade igual ou superior a 80 anos, sendo, $0,83 \%$ 
(65) octogenários, 1,11\% (88) nonagenários e 0,04\% (3) centenários.

Foram incluídos no estudo os idosos com idade igual ou superior a 80 anos que aceitaram participar da pesquisa através da assinatura do Termo de Consentimento Livre e Esclarecido (TCLE); em condições físico-funcionais de deambulação, com ou sem auxílio de dispositivos; e com condições de realizar o movimento de preensão manual.

Os idosos foram acessados em seus domicílios com o auxílio dos Agentes Comunitários de Saúde, no período de janeiro a fevereiro de 2014. As variáveis de interesse foram obtidas através de entrevista (anamnese), que abordou questões referentes a dados sociodemográficos (idade, sexo e escolaridade), presença de comorbidades e doenças, uso de medicamentos e quedas no último ano; exame físico-funcional, com avaliação do estado nutricional, velocidade da marcha e força de preensão manual; e aplicação de protocolos de identificação dos níveis de atividade física e fadiga, complementando a identificação de fenótipo de fragilidade.

A síndrome da fragilidade foi identificada de acordo com os cinco critérios propostos por Fried et al. (2001): 1) Perda de peso não intencional; 2) Exaustão avaliada por autorrelato de fadiga; 3) Diminuição da força de preensão manual; 4) Baixo nível de atividade física; e 5) Diminuição da velocidade da marcha.

'A perda de peso não intencional no último ano' foi verificada por meio do autorrelato, e, quando o idoso era portador da carteira de acompanhamento, essa informação era confirmada. Considerou-se como critério de fragilidade a perda de peso: $\geq 4,5 \mathrm{~kg}$ ou $\geq 5 \%$ do peso corporal no ano anterior.

'Exaustão avaliada por autorrelato de fadiga' foi definida através de questionamento extraído da CES-D (Center for Epidemiological Studies - Depression) (RADLOFF, 1977) a partir das seguintes perguntas: (1) sente que teve que fazer esforço para fazer tarefas habituais?; (2) não consegue levar adiante as suas atividades? Se o idoso respondeu na 'maioria das vezes e/ou sempre' para uma das duas questões, foi considerado como critério de fragilidade.

'Diminuição da Força de preensão manual' foi definida pelo teste de força de preensão manual, usando o Dinamômetro (Kratos ${ }^{\circledR}$ ). A baixa força muscular foi definida de acordo com sexo e índice de massa corporal (IMC) (SISVAN, 2004). Foi considerado critério de fragilidade os idosos que apresentaram resultados localizados entre $20 \%$ da média de três medidas (NERI; GUARIENTO, 2011).

'O baixo nível de atividade física' foi identificado com base em itens do Minnesota Leisure Time Actvity Questionnaire (TAYLOR ET AL., 1978). Foram considerados idosos ativos aqueles que acumularam pelo menos 120 minutos semanais em exercícios físicos e esportes ativos de intensidade vigorosa $(>6$ MET) ou aqueles com mais de 150 minutos semanais em exercícios físicos e esportes ativos de intensidade moderada (de $\geq 3 \mathrm{MET}$ $\mathrm{a} \leq 6 \mathrm{MET}$ ).

'Diminuição da velocidade da marcha' foi mensurada por meio do desempenho físico no teste de caminhada de 4,6 metros, cronometrado pelo tempo em segundos que cada idoso levou para concluir o percurso, em passo usual (GURALNICK ET AL., 1994). Os pontos de corte foram ajustados pelo sexo e pela altura: para homens com altura maior que 1,73 metros e mulheres com mais de 1,59 metros, consideraram-se frágeis os que realizaram o teste em tempo superior a seis segundos; e idosos com altura menor ou igual à especificada, quando completaram o teste em tempo superior a sete segundos.

A partir dos dados obtidos na avaliação, os idosos foram categorizados, de acordo com a classificação de Fried et al. (2001), em Frágil, Pré-frágil e Não frágil. No estágio Frágil, o idoso apresentou três ou mais componentes do fenótipo; no estágio Pré-frágil, apresentou um ou dois componentes do fenótipo; e Não frágil quando o idoso não apresentou nenhum dos componentes. 
Os dados obtidos foram analisados por meio do software Statistical Package for the Social Sciences (SPSS) (versão 18.0). Foram utilizadas ferramentas da estatística descritiva e analítica. Para a estatística descritiva, utilizaram-se medidas de tendência central, de dispersão e variabilidade. Para verificar a dependência e a associação de variáveis, $o$ teste do Qui-quadrado. Para todos os casos, foram considerados fatores significativos os valores correspondentes a $\mathrm{p}<0,05$.

Foram observados todos os aspectos éticos preconizados para pesquisas com seres humanos, com aprovação pelo Comitê de Ética em Pesquisa sob o parecer consubstanciado ${ }^{\circ} 615.533 / 2014$.

\section{Resultados}

Foram avaliados 69 idosos. Desses, todos apresentaram condição de fragilização: $42 \%$ foram classificados como pré-frágeis e $58 \%$ como frágeis. A maioria era do sexo feminino $(62,3 \%)$, e a média de idade foi de 85,0 $\pm 5,6$ anos (IC 95\% 83,7-86,44); idade mínima de 80 anos e máxima de 101. Quando relacionada à condição de fragilidade com as características sociodemográficas e as condições de saúde, não foi evidenciada diferença estatisticamente significativa entre idosos pré-frágeis e frágeis, de acordo com a tabela 1.

Tabela 1. Características sociodemográficas e condições de saúde em idosos longevos pré-frágeis e frágeis, Erval Seco (RS), 2014

\begin{tabular}{|c|c|c|c|c|}
\hline \multirow{2}{*}{ Variáveis } & & Pré-frágil $n=29$ & Frágil $n=40$ & \multirow{2}{*}{$\mathrm{p}$} \\
\hline & & $n(\%)$ & n (\%) & \\
\hline \multirow{2}{*}{ Sexo } & Masculino & $12(17,4)$ & $14(20,3)$ & \multirow{2}{*}{0,386} \\
\hline & Feminino & $17(24,6)$ & $26(37,7)$ & \\
\hline \multirow{3}{*}{ Faixa etária } & Octogenários & $25(36,2)$ & $32(46,4)$ & \multirow{3}{*}{0,32} \\
\hline & Nonagenários & $2(2,9)$ & $7(10,1)$ & \\
\hline & Centenários & $2(2,9)$ & $1(1,4)$ & \\
\hline \multirow{3}{*}{ Escolaridade } & Não frequentaram & $7(10,1)$ & $11(15,9)$ & \multirow{3}{*}{0,834} \\
\hline & $1-4$ anos & $18(26,1)$ & $22(31,9)$ & \\
\hline & 5 ou mais anos & $4(5,8)$ & $7(10,1)$ & \\
\hline \multirow{2}{*}{$\begin{array}{l}\text { Presença de doença } \\
\text { ou comorbidades }\end{array}$} & Sim & $26(37,7)$ & $36(52,2)$ & \multirow{2}{*}{0,632} \\
\hline & Não & $3(4,3)$ & $4(5,8)$ & \\
\hline \multirow{2}{*}{ Uso de medicamentos } & $\operatorname{Sim}$ & $24(34,8)$ & $38(55,1)$ & \multirow{2}{*}{0,096} \\
\hline & Não & $5(7,2)$ & $2(2,9)$ & \\
\hline \multirow{2}{*}{ Quedas no último ano } & $\operatorname{Sim}$ & $9(13,0)$ & $21(30,5)$ & \multirow{2}{*}{0,076} \\
\hline & Não & $20(29,0)$ & $19(27,5)$ & \\
\hline
\end{tabular}

Nota: Teste do Qui-quadrado com valores significativos correspondentes a $p<0,05$. 
A maior parte dos idosos do estudo apresentou baixa escolaridade formal; $26,1 \%$ dos idosos não frequentaram a escola e $73,9 \%$ relataram ter frequentado, no máximo, oito anos. Com relação à presença de doenças ou comorbidades, foi identificado que $91,3 \%$ dos idosos tinham, no mínimo, duas condições crônicas e, no máximo, cinco; a hipertensão arterial sistêmica (HAS) (71\%) foi a mais prevalente, seguida do diabetes mellitus (DM) $(36,2 \%)$, dislipidemia $(23,2 \%)$ e perda visual (18,8\%); outras doenças e comorbidades apresentaram menos de $10 \%$ de prevalência nessa população.

Ao inquirir sobre a ocorrência de quedas no último ano, evidenciou-se que $43,5 \%$ dos idosos sofreram pelo menos uma queda, com média de 2,5 $\pm 1,2$ quedas por idoso (IC $95 \%$ 0,73 -1,45); o número máximo de quedas relatadas foi seis.

Entre os componentes da fragilidade, a diminuição da velocidade da marcha esteve presente em $100 \%$ da amostra, seguida pela perda de peso (66,7\%), fadiga muscular $(65,2 \%)$, inatividade física $(23,2 \%)$ e redução da força de preensão manual (4,3\%). $\mathrm{O}$ número de componentes de fragilidade variou de um a quatro, com média de 2,59 \pm 0,8 componentes por idoso. A distribuição e a análise dos componentes de fragilidade entre idosos pré-frágeis e frágeis estão representadas na tabela 2.

Tabela 2. Frequência dos indicadores de fragilidade em idosos longevos pré-frágeis e frágeis, Erval Seco (RS), 2014

\begin{tabular}{|c|c|c|c|c|}
\hline Frequência dos indicadores de fragilidade & & Pré-frágil $29(42 \%)$ & Frágil $40(58 \%)$ & p \\
\hline \multirow{2}{*}{ Fadiga } & $\operatorname{Sim}$ & $12(17,4)$ & $33(47,8)$ & \multirow{2}{*}{$0,001^{*}$} \\
\hline & Não & $17(24,6)$ & $7(10,1)$ & \\
\hline \multirow{2}{*}{ Perda de peso } & $\operatorname{Sim}$ & $8(11,6)$ & $38(55,1)$ & \multirow{2}{*}{$0,000^{*}$} \\
\hline & Não & $21(30,4)$ & $2(2,9)$ & \\
\hline \multirow{2}{*}{ FPM } & $\operatorname{Sim}$ & $0(0)$ & $3(4,3)$ & \multirow{2}{*}{0,132} \\
\hline & Não & $29(42,0)$ & $37(53,6)$ & \\
\hline \multirow{2}{*}{ VM } & Sim & $29(42)$ & $40(58)$ & \multirow{2}{*}{-} \\
\hline & Não & $0(0)$ & $0(0)$ & \\
\hline \multirow{2}{*}{ Gasto energético } & Sim & $1(1,4)$ & $15(21,7)$ & \multirow{2}{*}{$0,001^{\star}$} \\
\hline & Não & $28(40,6)$ & $25(36,2)$ & \\
\hline
\end{tabular}

FPM: força de preensão manual; VM: velocidade de marcha; *: $p<0,05$.

Nota: Teste do Qui-quadrado.

Quanto às variáveis relacionadas aos indicadores de fragilidade, a média do IMC dos idosos foi de 24,9 $9 \pm 4,6$ (IC 95\% 23,8-26,0). O peso adequado foi encontrado em $49,3 \%$ dos idosos: entre as mulheres, $44,6 \%$, e entre os homens, $57,7 \%$; e o baixo peso foi encontrado em $26,1 \%$ dos idosos: entre as mulheres, $25,6 \%$, e entre os homens, $26,9 \%$. Com relação à velocidade da marcha, a média encontrada foi de 17,9 $\pm 6,3$ (IC 95\% 16,4-19,4); a força de preensão manual dos idosos avaliados foi de 1,27 $\pm 0,56$ (IC $95 \%$ 1,141,41); e o gasto energético (METS) foi de 6,2 $\pm 4,7$ (IC 5,0-7,3).

Na tabela 3, estão apresentados os componentes da fragilidade segundo sexo, e verificou-se maior prevalência, em ambos os sexos, de diminuição de velocidade de marcha, perda de peso e fadiga. A maioria apresentava um somatório de três componentes associados. 
Tabela 3. Prevalência dos indicadores de fragilidade por sexo em idosos longevos, Erval Seco (RS), 2014

\begin{tabular}{lrrr}
\hline \multirow{2}{*}{ Frequência dos indicadores de fragilidade } & Mulheres $\mathbf{n = 4 3}$ & Homens $\mathbf{n = 2 6}$ & População Geral $\mathbf{n = 6 9}$ \\
\cline { 2 - 4 } VM & $\mathbf{n}(\%)$ & $\mathbf{n}(\%)$ & $\mathbf{n}(\%)$ \\
Perda de peso & $43(100)$ & $26(100)$ & $69(100)$ \\
Fadiga & $31(72,1)$ & $15(57,7)$ & $46(66,7)$ \\
Gasto energético & $29(67,4)$ & $16(61,5)$ & $45(65,2)$ \\
FPM & $8(18,6)$ & $8(30,8)$ & $3(4,3)$ \\
\hline Número de componentes da fragilidade presentes & $2(4,6)$ & $1(3,8)$ & $8(11,6)$ \\
\hline 1 & $3(6,9)$ & $5(19,2)$ & $20(29,0)$ \\
2 & $13(30,2)$ & $7(26,9)$ & $33(47,8)$ \\
3 & $23(53,5)$ & $10(3,8)$ & $8(11,6)$ \\
\hline
\end{tabular}

VM: velocidade de marcha; FPM: força de preensão manual.

Nota: Valores apresentados em frequências absolutas e relativas

\section{Discussão}

Neste estudo, evidenciou-se que todos os idosos avaliados apresentavam condição de pré-fragilidade ou já encontravam-se frágeis. Destaca-se que a síndrome de fragilidade é uma condição relacionada a modificações fisiológicas, fatores sociodemográficos, psicológicos, nutricionais e à presença de comorbidades. Mello, Engstrom e Alves (2014), em uma revisão sistemática, identificaram como fatores associados à fragilidade o aumento da idade, o sexo feminino, a escolaridade, o número de doenças, a presença de doença cardiovascular, a incapacidade funcional, a avaliação ruim de saúde, a depressão, o declínio cognitivo e outros. Dados semelhantes foram encontrados no estudo longitudinal de Hajek et al. (2016), realizado na Alemanha, com idosos acima de 80 anos, no qual o aumento da idade esteve associado tanto à fragilidade geral como ao sexo masculino e ao feminino. Os dados deste estudo diferem dos estudos supracitados e podem estar relacionados às características desta população: de idosos residentes em um município de pequeno porte que reserva traços de estilo de vida rural.

A prevalência do sexo feminino está de acordo com a literatura (MELLO; ENGSTROM; ALVES, 2014; DUARTE; PAÚL, 2015; FRIED ET AL., 2001), Uma veZ que a feminização da população é um processo evidenciado atualmente na sociedade brasileira. Além disso, a prevalência da fragilidade no sexo feminino é semelhante ao que demonstram tanto as pesquisas nacionais (PEGORARI; TAVARES, 2014; SANTOS ET AL., 2015) quanto internacionais (JÜRSCHIK ET AL., 2012; BUTTERY ET AL., 2015). Tal fato pode ser explicado pela maior perda fisiológica de massa muscular no sexo feminino com o envelhecimento, além de as mulheres serem mais propensas ao desenvolvimento de sarcopenia, que é um risco intrínseco para o desenvolvimento da fragilidade (ESPINOZA; FRIED, 2007), que agrava-se pela sobrecarga de doenças (FRIED ET AL., 2001).

Com relação à escolaridade, observou-se no presente estudo que $26,1 \%$ dos idosos participantes são analfabetos e que os demais frequentaram a escola por, no máximo, oito anos. A baixa escolaridade foi semelhante à identificada por Pegorari e Tavares (2014) 
entre idosos residentes em Uberaba (MG), e por Bazzanella, Picolli e Quevedo (2015) com um grupo de convivência de Caxias do Sul (RS). Destaca-se que a escolaridade não atua diretamente na fisiopatologia da fragilidade, porém, interfere no estilo e na qualidade de vida do idoso e, consequentemente, pode influenciar o processo de fragilização $(\mathrm{HIRSCH}$ ET AL., 2006). As revisões de Mello, Engstrom e Alves (2014) e de Pegorari e Tavares (2014) apresentam a baixa escolaridade como um fator associado à fragilidade. Apesar de a baixa escolaridade também ter sido evidenciada na população estudada nesta pesquisa, não se identificou diferença estatisticamente significativa entre escolaridade e fragilidade.

Das doenças e comorbidades de maior prevalência, este estudo evidenciou a HAS e DM. Esses achados são semelhantes aos de Pegorari e Tavares (2014) e Freitas et al. (2016), que verificaram a presença de doença ou comorbidade em $97,8 \%$ e $100 \%$ dos idosos, respectivamente. Santos et al. (2015) identificaram a prevalência de HAS em $62,5 \%$ e de DM em $15,1 \%$. Destaca-se que a presença de doenças cardiovasculares e comorbidades é frequente em idades mais avançadas, as quais podem tornar o idoso mais vulnerável à fragilidade pelas limitações físicas relacionadas a elas (FRIED; WALSTON, 2003). A maioria dos idosos referiu o uso contínuo de medicamentos, o que vai ao encontro de estudos realizados com a população idosa brasileira (DAL PIZZOL ET AL., 2012; PEREIRA ET AL., 2015).

A ocorrência de queda no último ano foi relatada por $43,5 \%$ dos idosos no presente estudo. Santos et al. (2015) e Vieira et al. (2013) verificaram a ocorrência de quedas em $27,5 \%$ e $28,0 \%$ dos idosos, respectivamente. No entanto, em ambos, a idade média foi inferior à dos idosos longevos, fator esse que pode ter influenciado para a maior prevalência no presente estudo.

A presença de fragilidade no idoso varia de acordo com a faixa etária, o local do estudo e os critérios adotados para a sua avaliação. Em pesquisas nacionais, Vieira et al.
(2013), Pegorari e Tavares (2014) e Santos et al. (2015) verificaram a presença de fragilidade ou pré-fragilidade em $55,0 \%, 67,3 \%$ e $78,5 \%$, respectivamente.

Em estudo realizado na Alemanha, com população com idade entre 65 a 79 anos, identificou-se a prevalência de fragilidade em 2,6\% dos idosos estudados, e 38,8\% foram classificados como pré-frágeis, entre os quais, $2,3 \%$ dos homens eram frágeis e $36,9 \%$ pré-frágeis; enquanto $2,8 \%$ das mulheres eram frágeis e 40,4\% pré-frágeis. Segundo Duarte e Paúl (2015), em estudo realizado em Portugal com idosos divididos em grupos etários (50-64; 65-74 e $\geq 75)$, denota-se que as percentagens de fragilidade estão mais presentes à medida que a idade avança. Dessa forma, os autores destacam que não existem pessoas com 75 anos ou mais na condição de não frágeis, sendo esse grupo etário dividido entre pré-frágil (37,7\%) e frágil (60,4\%), ou seja, mais de metade da população estudada com mais de 75 anos de idade foi considerada frágil. Os resultados da pesquisa aqui apresentada corroboram os estudos citados, mas há de se ponderar que a população brasileira tem características de envelhecimento bastante diferenciadas das dos idosos europeus, dada a qualidade de vida destes e, também, as políticas públicas mais avançadas, que estimulam promoção e prevenção. Consequentemente, envelhece-se com uma menor carga de doenças.

No tocante aos critérios de avaliação da síndrome da fragilidade, a diminuição da velocidade da marcha está presente em $100 \%$ dos idosos longevos estudados. Semelhantemente ao verificado por Kan et al. (2009), em que, entre os preditores de incapacidade, a redução da velocidade da marcha foi verificada em $80 \%$ dos idosos frágeis.

Nos achados deste estudo, evidenciou-se que a maioria dos idosos sofreu perda de peso não intencional no último ano. A associação de fragilidade com baixo peso pode ter relação com a perda de massa muscular comum em indivíduos em processo de 
emagrecimento não intencional (CHEMIN; MURA, 2007). A perda de peso não intencional parece surgir em decorrência da desregulação energética gerada por alterações neuroendócrinas e musculoesqueléticas, desnutrição, inflamação, doenças catabólicas e diminuição da massa muscular (FRIED; WALSTON, 2003).

Vieira et al. (2013) identificaram que o baixo nível de atividade física e a lentidão na velocidade de marcha foram os dois critérios de fragilidade mais frequentes entre os idosos pré-frágeis e frágeis e constataram, também, que os idosos frágeis apresentavam limitações nas atividades instrumentais da vida diária (AIVD), que incluem tarefas como banhar-se, vestir-se, alimentar-se, usar o sanitário, realizar transferências e continência; e restrição nas atividades avançadas de vida diária (AAVD), tais como lazer, trabalho e engajamento social. Do mesmo modo, Bazzanela, Picolli e Quevedo (2015), ao verificarem os níveis de qualidade de vida percebida e de atividade física de idosas acima de 80 anos, constataram um baixo envolvimento das idosas avaliadas na prática de atividades físicas, concentrando-se tal prática nas lidas domésticas, entre as quais, $79,3 \%$ das idosas foram classificadas como insuficientemente ativas. Em contrapartida, o presente estudo demonstrou, com relação ao nível de atividade física, que a grande maioria dos idosos longevos é ativa (76,8\%), dadas as suas características.

O envelhecimento com fragilidade torna o idoso mais vulnerável e com baixa capacidade para suportar fatores de estresse, o que resulta na maior probabilidade de adoecer e, consequentemente, em um elevado número de hospitalizações, que leva a uma maior dependência (PEREIRA ET AL., 2015). Isso demonstra a necessidade de os profissionais de saúde estarem habilitados a atuar com esse segmento etário em expansão, considerando a singularidade do envelhecimento de cada pessoa e a repercussão positiva no cuidado, com vistas à manutenção da autonomia do indivíduo idoso (CUNHA ET AL., 2012). Entretanto, embora o envelhecimento possa predispor-se à síndrome de fragilidade, nem todos os idosos são frágeis ou pré-frágeis, o que sugere que essa síndrome possa se constituir em características mais acentuadas do que as do processo normativo de envelhecimento fisiológico (FULOP ET AL., 2010).

\section{Conclusões}

Destaca-se que a maioria dos idosos longevos está em condição de fragilidade; e que, entre os componentes que integram essa condição, a redução da velocidade da marcha, a perda de peso e a fadiga foram os mais frequentes. Os resultados deste estudo mostram a prevalência do sexo feminino, a baixa escolaridade, a presença de doenças crônicas e a ocorrência frequente de quedas na população de idosos.

Dessa forma, é evidente a fragilização de idosos com idade igual ou superior a 80 anos. A identificação da prevalência de fragilidade deve subsidiar a proposição de políticas públicas para essa parcela da população, com tendência de crescimento nos próximos anos. Deve, também, subsidiar o planejamento das ações de saúde em todos os níveis de atenção na busca de garantir a integralidade da atenção, o apoio e o suporte social ao idoso e à sua família para uma velhice digna.

A avaliação e a classificação dos idosos de acordo com os critérios de fragilidade possibilitam organizar as prioridades de assistência, especialmente na Atenção Primária, com o objetivo de prevenir a fragilidade, manter e/ou restaurar a capacidade funcional e a autonomia. 


\section{Referências}

BAZZANELLA, N. A. L.; PICOLLI, J. C. J.; QUEVEDO,

D. M. Qualidade de vida percebida e atividade física: um estudo em idosas acima de 80 anos participantes de um programa municipal de saúde da terceira idade na Serra Gaúcha, RS. Estudos interdisciplinares sobre Envelhecimento, Porto Alegre, v. 20, n. 1, p. 249-270, 2015.

BRASIL. Ministério de Saúde. Departamento de Informática do Sistema Único de Saúde. 2012. Tabnet. Disponível em: <http://tabnet.datasus.gov.br/cgi/deftohtm.exe?ibge/cnv/popuf.def>. Acesso em: 10 abr. 2016

BUTTERY, A. K. et al. Prevalence and correlates of frailty among older adults: findings from the German health interview and examination survey. BMC Geriatrics, Londres. v. 15, n. 22, p. 1-9, 2015.

CHEMIN, S. S.; MURA, J. D. P. Tratado de alimentação, nutrição e dietoterapia. São Paulo: Roca, 2007.

CUNHA, J. X. P. et al. Autonomia do idoso e suas implicações éticas na assistência de enfermagem. Saúde em Debate, Rio de Janeiro, v. 36, n. 95, p. 657-664, 2012.

DAL PIZZOL, T. S. et al. Uso de medicamentos entre idosos residentes em áreas urbanas e rurais de município no Sul do Brasil: um estudo de base populacional. Cadernos de Saúde Pública, Rio de Janeiro, v. 28, n. 1, p. 104-114, 2012.

DUARTE, M.; PAÚL, C. Prevalência de fragilidade fenotípica em pessoas em processo de envelhecimento numa comunidade portuguesa. Revista Brasileira de Geriatria e Gerontologia, Rio de Janeiro, v. 18, n. 4, p. 871-880, 2015

ESPINOZA, S. E.; FRIED, L. P. Risk factors for frailty in the older adult. Clinical Geriatrics, v. 15, n. 6, p. 37-44, 2007.

FREITAS, C. V. et al. Avaliação de fragilidade, capacidade funcional e qualidade de vida dos idosos atendidos no ambulatório de geriatria de um hospital universitário. Revista Brasileira de Geriatria e Gerontologia,
Rio de Janeiro, v. 19, n.1, p. 119-128, 2016.

FRIED, L. P.; WALSTON, J. M. Frailty and failure to thrive. In: HAZARD, W. R. et al. Principles of Geriatric Medicine and Gerontology. 5. ed. Nova Iorque: McGrawHill, 2003. p. 1487-1502.

FRIED, L. P. et al. Frailty in older adults: evidence for a phenotype. Journals of Gerontology Series A: Biological Sciences and Medical Sciences, Washington, DC, v. 56, n. 3, p. 146-156, 2001.

FRIED, L. P. et al. Untangling the concepts of disablity, frailty and comorbidity: implications for improved targeting and care. Journals of Gerontology Series A: Biological Sciences and Medical Sciences, Washington, DC, v. 59, n. 3, p. 255-63, 2004.

FULOP, T. et al. Aging, frailty and age-related diseases. Biogerontology, Dordrecht, v. 11, n. 5, p. 547-63, 2010.

GURALNICK, J. M. et al. Short physical perfomance batery assessing lower extremity function association with self reported. Journal of Gerontology, St. Louis, v. 49, n. 2, p. 85- 94, 1994.

HAJEK, A. et al. Predictors of Frailty in Old Age Results of a longitudinal study. The Journal of Nutrition Health and Aging; Paris, v. 20, n. 9, p. 952-957, 2016.

HIRSCH, C. et al. The association of race with frailty: The Cardiovascular Health Study. Annals of Epidemiology, Nova Iorque, v. 16, n. 7, p. 545-53, 2006.

\section{INSTITUTO BRASILEIRO DE GEOGRAFIA E} ESTATÍSTICA (IBGE). Brasil, Rio Grande do Sul, Erval Seco: População. Disponível em: < https://cidades.ibge. gov.br/v4/brasil/rs/erval-seco/panorama>. Acesso em: 10 out. 2010

JURSCHIK, P. et al. Prevalence of frailty and factors associated with frailty in the elderly population of Lleida, Spain: The FRALLE survey. Archives of Gerontology and Geriatrics, v. 55, n. 3, p. 625-31, 2012. 
KALACHE, A. O mundo envelhece: é imperativo criar um pacto de solidariedade social. Ciência \& Saúde Coletiva, Rio de Janeiro, v. 13, n. 4, p. 1107-1111, 2008.

KAN, G. A. V. et al. Gait speed at usual pace as a predictor of adverse outcomes in commu-nity-dwelling older people an International Acade-my on Nutrition and Aging (IANA) Task Force. The Journal of Nutrition, Health \&t Aging, Paris, v. 13, n.10, p. 881-9, 2009.

MELLO, A. C.; ENGSTROM, E. M.; ALVES, L. C. Fatores sociodemográficos e de saúde associados à fragilidade em idosos: uma revisão sistemática de literatura. Cadernos de Saúde Pública, Rio de Janeiro, v. 30, n. 6, p.1-25, 2014.

NERI, A. L.; GUARIENTO, M. E. (Org.). Fragilidade, saúde e bem-estar em idosos: dados do estudo FIBRA Campinas. Campinas: Alínea, 2011. (Coleção Velhice e Sociedade).

PEGORARI, M. S.; TAVARES, D. M. S. Fatores associados à síndrome de fragilidade em idosos residentes em área urbana. Revista Latino-Americana de Enfermagem, Ribeirão Preto, v. 22, n. 5, p. 874-82, 2014.

PEREIRA, L. F. et al. Retrato do perfil de saúde-doença de idosos longevos usuários da atenção básica de saúde. Revista de Enfermagem da UERJ, Rio de Janeiro, v. 23, n. 5, p. 649-55, 2015.

RADLOFF, L. S. The CES-D Scale: A Self-Report depression scale for research in the general population. Applied Psychological Measurement. Champaign, Illinois, EUA, v. 1, p. 385-401, 1977.
SANTOS, P. H. S. et al. Perfil de fragilidade e fatores associados em idosos cadastrados em uma Unidade de Saúde da Família. Ciência \&t Saúde Coletiva, Rio de Janeiro, v. 20, n. 6, p. 1917-1924, 2015.

\section{SISTEMA VIGILÂNCIA ALIMENTAR E}

NUTRICIONAL (SISVAN). Orientações básicas para a coleta, processamento, análise de dados e informação em serviços de saúde. Brasília, DF: Ministério da Saúde, 2004.

TAYLOR, H. L. et al. A questionnaire for the assessment of leisure time physical activities. Journal of Chronic Diseases, St. Louis, v. 31, n. 12, p. 741-755, 1978.

TEIXEIRA, I. N. D. O.; NÉRI, A. L. A fragilidade no envelhecimento: fenômeno multidimensional, multideterminado e evolutivo. In: FREITAS, E. V. et al. Tratado de Geriatria e Gerontologia. 2. ed. Rio de Janeiro: Guanabara Koogan, 2006, p. 1102-9.

VERMEIREN, S. et al. Frailty and the Prediction of Negative Health Outcomes: A Meta-Analysis. Journal of the American Medical Directors Association, Hagerstown, v. 17, n. 12 p.1163.el-1163.e17, 2016.

VIEIRA, R. A. et al. Prevalência de fragilidade e fatores associados em idosos comunitários de Belo Horizonte, Minas Gerais, Brasil: dados do Estudo FIBRA.

Cadernos de Saúde Pública, Rio de Janeiro, v. 29, n. 8, p. $1631-1643,2013$

\footnotetext{
Recebido para publicação em dezembro de 2016

Versão final em maio de 2017

Conflito de interesses: inexistente

Suporte financeiro: não houve
} 